\title{
High expression of kinesin light chain-2, a novel target of miR-125b, is associated with poor clinical outcome of elderly non-small-cell lung cancer patients
}

\author{
M Wang ${ }^{1,2}, X_{\text {Zhu }}{ }^{* 1,2}$, Z Sha ${ }^{1}, \mathrm{~N} \mathrm{Li}^{1}, \mathrm{D} \mathrm{Li}{ }^{1}$ and L Chen ${ }^{*, 1}$ \\ ${ }^{1}$ Department of Radiation Oncology, Nanfang Hospital, Southern Medical University, Guangzhou, Republic of China
}

Background: MiR-125b has critical role in non-small-cell lung cancer (NSCLC) cell migration, and its target genes have not been elucidated. Kinesin-1 light chain (KLC)-2 was predicted as one of miR-125b's targets by bioinformatics analysis. This study is to identify the function of KLC2 and its interaction with miR-125b in NSCLC.

Methods: Kinesin-1 light chain-2 protein expression and its clinical relevance were analysed in 140 matched NSCLC and adjacent non-neoplastic lung tissues. Both KLC2 gain- and loss-of-function analyses were performed in NSCLC cell lines by transient transfection. The direct interaction between KLC2 and miR-125b was confirmed by a luciferase reporter assay and a transient cotransfection assay as well as an analysis of eight matched clinical samples.

Results: KLC2 protein was upregulated in NSCLC cell lines and tissues, and was an independent predictor of poor prognosis for elderly NSCLC patients. Kinesin-1 light chain-2 remarkably enhanced the invasive and migratory ability of NSCLC cells. MiR-125b inhibited KLC2 3'-untranslated region luciferase activity and protein expression, and inversely correlated with KLC2 expression in clinical samples. Kinesin-1 light chain-2 almost completely reversed miR-125b-induced inhibition on migration and invasion.

Conclusions: Kinesin-1 light chain-2 protein overexpression predicts poor survival in elderly NSCLC patients. Kinesin-1 light chain2 acts as a proto-oncogene and a functional target of miR-125b in NSCLC cells.

Lung cancer is estimated to be responsible for nearly one-fifth of cancer-related deaths worldwide in 2012 (Ferlay et al, 2013). Metastasis is still the leading cause of failure in clinical management of lung cancer. Increasing evidence supports the involvement of microRNAs in the development of many human cancers including lung cancer. Recently, miR-125b has been demonstrated to have important roles in the invasion and migration and its reported targets differed in various cancers. For example, miR$125 \mathrm{~b}$ could inhibited melanoma cells migration by direct regulation of C-Jun protein expression (Kappelmann et al, 2013) or targeting serine/threonine kinase mixed lineage kinase- 3 at both the transcriptional and translational levels (Zhang et al, 2014).
MiR-125b was also reported ( $\mathrm{Li}$ et al, 2014) to function as a tumour suppressor and inhibit Ewing's sarcoma cell migration and invasion by targeting the $\mathrm{PI} 3 \mathrm{~K} / \mathrm{Akt} / \mathrm{mTOR}$ signalling pathway. MiR-125b exerted tumour-suppressive effects in hepatocellular carcinoma metastasis not only through the suppression of oncogene Lin28 homologue B (LIN28B) expression (Liang et al, 2010) but also by the downregulation of suppressor of variegation 3-9 homologue 1 (SUV39H1; Fan et al, 2013). In addition, matrix metallopeptidase-13 was identified as a direct target suppressed by miR-125b in cutaneous squamous cell carcinoma (Xu et al, 2012). Contrarily, the role of miR-125b in pro-metastasis was verified both in breast cancer cells and type II endometrial carcinoma cells

\footnotetext{
${ }^{\star}$ Correspondence: Dr X Zhu; E-mail: zhuxx01@126.com or Professor L Chen; E-mail: chenlonghuafl@126.com

${ }^{2}$ These authors contributed equally to this work.
} 
by targeting tumour-suppressor genes, StAR-related lipid transfer domain containing-13 and tumour protein 53-induced nuclear protein 1 separately (Jiang et al, 2011; Tang et al, 2012). In lung cancer, patients with high serum miR-125b expression displayed a significantly poorer prognosis (Yuxia et al, 2012). In our previous studies (Li et al, 2013), we identified miR-125b as a downregulated miRNA in non-small-cell lung cancer (NSCLC) cell line upon metastasis-associated gene- 1 depletion and verified that miR-125b reversed the stimulatous effect of metastasis-associated gene- 1 on the migration and invasion. However, the target genes elucidating the role of miR-125b in NSCLC invasion and migration have not been identified.

Recently, we used bioinformatics analysis to identify its target genes. Kinesin-1 light chain (KLC)-2 was identified as one of the candidate targets of miR-125b based on bioinformatics analysis from three publicly available miRNA databases (Targetscan, miRanda, miRWalk), potential binding site to miR-125b was predicted in the $3^{\prime}$-untranslated region ( $\left.3^{\prime} \mathrm{UTR}\right)$ of KLC2.

KLC2 is one of the best characterised light chain isoforms of Kinesin-1, a widely expressed molecular motor that drives transport of numerous cargoes including intracellular organelle and a variety of vesicle subtypes along microtubules (Hirokawa et al, 2009). Smad2, required for transduction of TGF- $\beta$ signals, is a known KLC2-binding partner and kinesin-1 cargo (Batut et al, 2007). TGF- $\beta$ signalling have a pleiotropic action during tumorigenesis (Akhurst and Hata, 2012). Another clue to KLC2 function in cancer comes from the findings that KLC2 was involved in the regulation of lemur tyrosine kinase-2, a susceptibility gene for prostate cancer, on TGF- $\beta$-induced Smad2 signalling (Manser et al, 2012).

In the present study, we evaluated the clinical significance of KLC2 in NSCLC and its effect on the malignant phenotype of NSCLC cells. Next, we identified KLC2 as a novel target of miR-125b in NSCLC and confirmed the direct interaction between miR-125b and KLC2.

\section{MATERIALS AND METHODS}

Tissue samples and chips. Sixteen fresh tissues including primary NSCLC $(n=8)$ and adjacent non-tumour lung $(n=8)$ were obtained from the Cardiothoracic Surgery of Nanfang Hospital (Southern Medical University, Guangzhou, China). None of the patients had received chemotherapy or radiotherapy before surgery. Our study protocol was approved by the Ethics Review Board of Nanfang Hospital. Fresh tissues were acquired with written informed consent of each patient. Both normal and tumour tissues were histologically confirmed in the Nanfang Hospital Department of Pathology.

Tissue chips consisted of 140 pairs of human primary NSCLC and their adjacent non-neoplastic lung tissues were obtained from Outdo Biotech CO. Ltd. (Shanghai, China).

Cell cultures. Human NSCLC cell lines (SPC-A-1, 95D, A549, H460 and H1299) were purchased from the Shanghai Cell Bank of Chinese Academy of Science (Shanghai, China) and maintained in complete medium with high-glucose RPMI-DMEM (Hyclone, GE Healthcare Life Sciences, Piscataway, NJ, USA) and 10\% fetal bovine serum (FBS, Gibco, Grand Island, NY, USA). An immortalised bronchial epithelial cell HBE and 293T cell were cultured in RPMI-1640 (Hyclone) with 10\% FBS. All cells were incubated at $37^{\circ} \mathrm{C}$ in a humidified atmosphere containing $5 \% \mathrm{CO}_{2}$.

Plasmid and oligonucleotide construction. KLC2 (http://www. ncbi.nlm.nih.gov/nuccore/NM_022822)-coding sequence, which do not contain the predicted miR-125b-binding sites, was cloned into pReceive-M98 vector (synthesised by GeneCopoeia, Rockville, MD, USA). Short hairpin RNA sequence targeting KLC2 gene of human was inserted into hU6-MCS-Ubiquitin-EGFP-IRES-puromycin vector to generate KLC2-si plasmid, and plasmid transfected with scramble siRNA ( $5^{\prime}$-TTCTCCGAACGTGTCACGT-3') was used as KLC2-si/control. The sequence was KLC2-si: sense, $5^{\prime}$-caG ATCCGCAAGTTGGATGAATTCATCCAACTTGCGGATCTG-3' (synthesised by Genechem, Shanghai, China). The miR-125b inhibitor, nonspecific control miRNA (inhibitor/NC), miR-125b mimics and negative control (mimics/NC) were designed and synthesised by Genepharma (Shanghai, China). The sequences were as follows, miR-125b inhibitor: $5^{\prime}$-UCACAAGUUAGGGUC UCAGGGA-3'; inhibitor/NC: 5'-CAGUACUUUUGUGUAGUAC AA-3'; miR-125b mimics: $5^{\prime}$-UCCCUGAGACCCUAACUUGUG AACAAGUUAGGGUCUCAGGGAUU-3'; mimics/NC: $5^{\prime}$-UUC UCCGAACGUGUCACGUTT-3'.

Transient transfection. Cell lines were seeded $\left(2 \times 10^{5}\right.$ per well $)$ onto six-well plate $18-24 \mathrm{~h}$ before transfection. Appropriate aforementioned plasmids ( $3 \mu \mathrm{g}$ per well), miR-125b inhibitor, mimics or NC (100 pmol) were added to corresponding wells using Lipofectamine 3000 reagent (Invitrogen, Shanghai, China). Cells were then incubated under $5 \% \mathrm{CO}_{2}$ at $37^{\circ} \mathrm{C}$ and harvested at $48 \mathrm{~h}$ post transfection. Co-transfection of KLC2 with miR-125b in SPC-A-1 and 95D cells were performed according to the similar steps.

RNA extraction and quantitative RT-PCR. Total RNAs of human tissues or cultured cells were extracted with Trizol reagent (Takara, Dalian, China) following the manufacturer's recommended protocol. cDNA was synthesised by using total RNAs according to the manufacturer's instruction (PrimeScript RT reagent Kit, TaKaRa, Dalian, China). Quantitative RT-PCR was undertaken in a LightCycler 480 real-time PCR system (Roche, Basel, Switzerland) with SYBR Premix ExTaq (TaKaRa). MiR-125b expression levels were normalised to U6 small nuclear RNA (U6 snRNA) as the internal control. For KLC2 mRNA analysis, GAPDH was used as an internal control. Both the prime sequences of miR-125b and KLC2 were synthesised by Invitrogen. The sequences of the primers are shown as follows, hsa-miR-125b forward: $5^{\prime}$-ACACTCCAGCTGGGTCCCTGAGACCCTAACT- ${ }^{\prime}$; U6 forward: $5^{\prime}$-GCTTCGGCAGCACATATACTAAAAT-3'. MiR universal reverse prime: $5^{\prime}$-CTCAACTGGTGTCGTGGA-3' (TaKaRa). Kinesin-1 light chain-2 forward: 5' -AGGGGATGTGTCTGGTCAG-3', reverse: $5^{\prime}$-CCTGTGAGGCGTATTGGATCA-3'. GAPDH forward: 5'-GCACCGTCAAGGCTGAGAAC-3', reverse: 5' -TGGTGAAGAC GCCAGTGGA- $3^{\prime}$. The relative expression of miR-125b and KLC2 was calculated according to the $2^{-\Delta \Delta C t}$ method.

Western blot analysis. Total protein from tissues and cells was extracted using RIPA lysis buffer. Western blot was performed as described previously (Zhu et al, 2010). Primary antibodies were as follows: rabbit polyclonal anti-KLC2 antibody ( $1: 1000$; Proteintech, Chicago, IL, USA; 17668-1-AP), mouse polyclonal anti- $\beta$ actin (1:2000; Santa Cruz, Santa Cruz, CA, USA; sc-130301), horseradish peroxidase-conjugated goat anti-rabbit IgG (1:5000; Ebiogenes, Guangzhou, China; ab136817), and horseradish peroxidase-conjugated goat anti-mouse IgG (1:5000; Ebiogenes; $\mathrm{ab} 136815)$. The anti- $\beta$-actin antibody was used as the loading control. Finally, the active bands were visualised and quantified by software QuantityOne v4.6.2.

Wound-healing assay. The trypsinized cells $\left(1 \times 10^{6}\right.$ per well $)$ were plated on six-well plates, cultured overnight in complete medium and transfected with corresponding plasmids or oligonucleotide aforementioned. Upon confluence reached approximately $90 \%$, the monolayers of cells were scratched using a sterile $10 \mu \mathrm{l}$ plastic pipette tip. Cells were washed with culture medium two times to remove cell debris and cultured again under $5 \% \mathrm{CO}_{2}$ at $37^{\circ} \mathrm{C}$ up to $48 \mathrm{~h}$ in serum-free medium. Images were captured under an inverted microscope following wounding ( 0 and $48 \mathrm{~h}$ ) to assess the rate of gap healing. 
Transwell migration and invasion assays. Transfected cells $\left(4 \times 10^{5}\right.$ per $\left.200 \mu \mathrm{l}\right)$ in serum-free medium were planted to the upper Transwell chamber (BD Bioscience, Bedford, MA, USA, 24-well insert, pore size: $8 \mu \mathrm{m})$. Complete medium $(500 \mu \mathrm{l})$ containing $10 \%$ FBS as a Chemoattractant was placed to the lower chambers. For the invasion assay, the surface of lower chamber was covered with Matrigel (BD Biosciences, Sparks, MD, USA). After $24-48 \mathrm{~h}$ of incubation under standard culture conditions, tumour cells were migrated or invaded through the bottom of membrane filter. After removing the cells on the top side of the membrane by wet cotton swab, cells that had migrated or invaded to the underside were fixed in methanol for $20 \mathrm{~min}$, then stained with $0.1 \%$ crystal violet solution for $30 \mathrm{~min}$ and washed with PBS finally. Five visual fields of each chamber were randomly chosen and photographed under the inverted microscope. The migrating or invading cells were counted offline and presented with the average value.

Dual-luciferase-reporter assay. The fragments containing the mutant-type (MUT) or wild-type (WT) putative target site of human KLC2 3'UTR were cloned into mirGLO vector plasmids (Genepharma). For the luciferase reporter assay, 293T or SPC-A-1 cells were co-transfected with $20 \mathrm{pmol}$ miR-125b mimics or the negative control and $0.5 \mu \mathrm{g}$ of mirGLO-KLC2-3'UTR-WT, mirGLO-KLC2-3'UTR-MUT using Lipofectamine 3000 reagent (Invitrogen). Cells were harvested at $48 \mathrm{~h}$ after co-transfection following the manufacturer's instructions, and luciferase activity was assayed with Dual-Luciferase Reporter Assay System

A
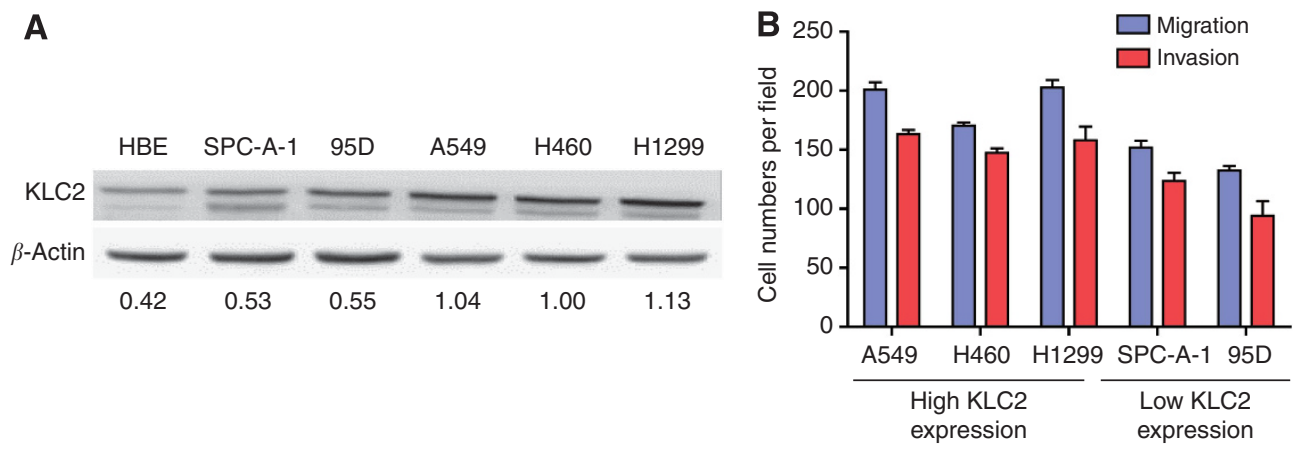

C
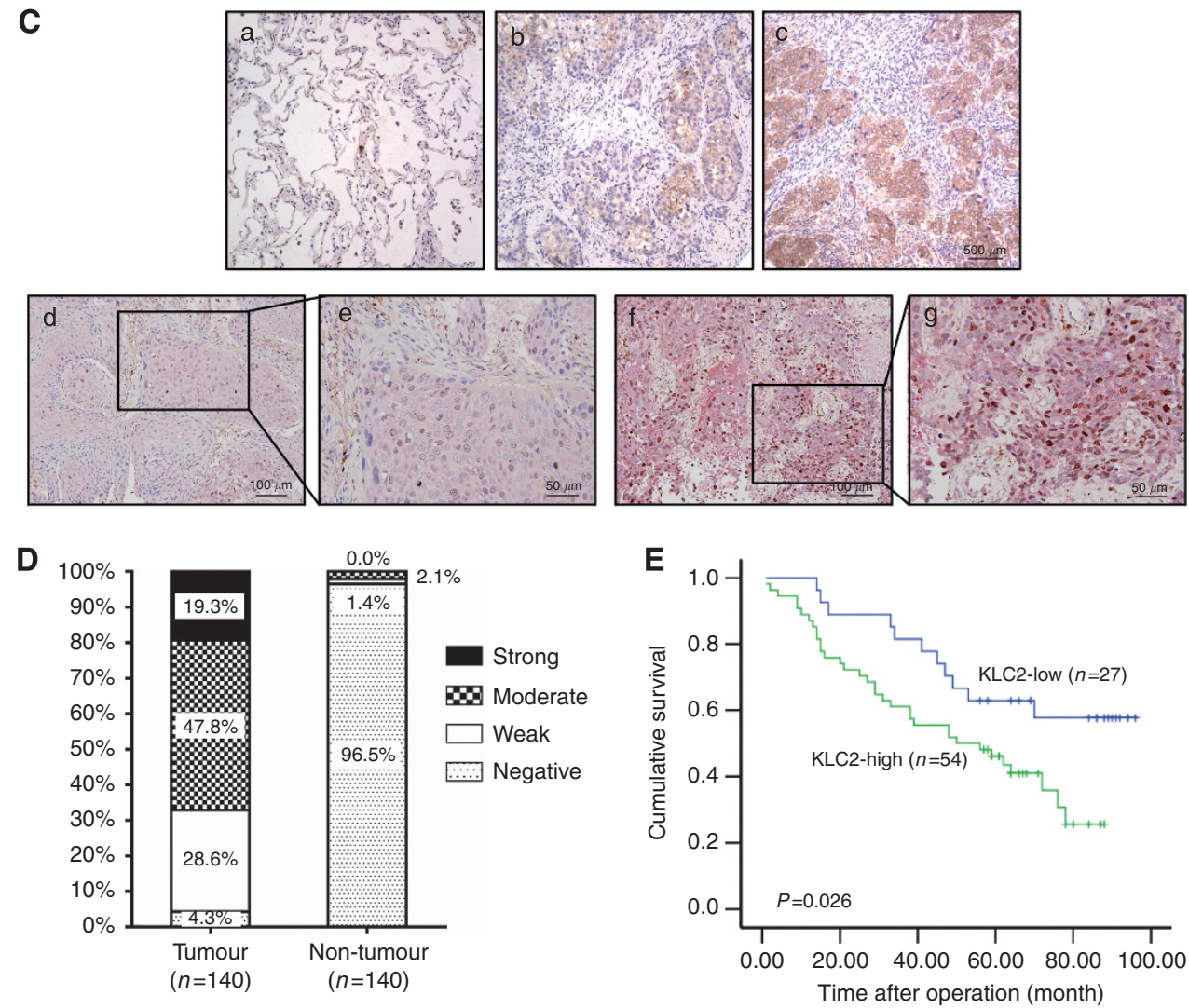

Figure 1. Expression of KLC2 protein in human NSCLC cell lines and tissue microarray samples. (A) Western blotting analysis of KLC2 protein levels in NSCLC cell lines and immortalised bronchial epithelial cell HBE. (B) Transwell analysis to determine the migration and invasion of A549, H460, H1299, SPC-A-1 and 95D cells. (C) Representive images of KLC2 staining in (a) adjacent non-neoplastic lung tissue $(\times 200)$, NSCLC clinical samples with (b) low expression of KLC2 ( $\times 200$ ) and (c) high expression of KLC2 ( $\times 200$ ). Double immunohistochemical staining of KLC2 (red colour cytoplasmic stain) and Ki-67 (brown colour nuclear stain) in NSCLC clinical samples (d and f, $\times 200$; e and g, $\times 400$ ). (D) Distribution of the proportion of different KLC2 expression levels in NSCLC tissues and non-tumour lung tissues. (E) Kaplan-Meier survival analysis of overall survival in elderly NSCLC patients ( $\geq 60$ years of age, $n=81$ ). 
(Promega, Madison, WI, USA). Finally, the ratio of firefly and Renilla (internal control) luciferase activities were calculated.

Immunohistochemical staining. Immunohistochemical staining was carried out with immunohistochemistry kit (Zhong ShanGolden Bridge Biological Technology, Beijing, China) according to the manufacturer's protocol. A rabbit polyclonal antibody for KLC2 (1:100; Proteintech; 17668-1-AP) was used as primary antibody. The cytoplasmic staining of KLC2 in tumour tissue was scored by an semi-quantitative method reported previously (Zhu et al, 2010). The intensity of protein expression was scored as following: $0=$ negative cytoplasmic staining, $1=$ intermediate brown cytoplasmic staining, $2=$ brown cytoplasmic staining and $3=$ dark brown cytoplasmic staining. The extent of protein expression was quantified by evaluating the percentage, scored as 0 (none), 1 (<10\%), $2(10 \%-50 \%), 3(51 \%-80 \%)$ and $4(<80 \%)$, of the positive stained areas in relation to the entire tumour areas on the slides. The final immunoreactive score was determined by multiplying the intensity score by the extent score, with the minimum score attainable being 0 and the maximum being 12 . The cutoff threshold was set as 4 and a score of $\geq 4$ was considered as high KLC2 expression and $<4$ as low KLC2 expression.

Double immunohistochemical staining was performed with polymer double staining kit $(\mathrm{Mo} / \mathrm{HRP}+\mathrm{Rb} / \mathrm{AP}$; Zhong Shan-Golden Bridge Biological Technology, Catalogue number: DS-0001) according to the manufacturer's protocol. Slides were washed in PBS for $5 \mathrm{~min}$ at room temperature and incubated with paraformaldehyde for $4 \mathrm{~min}$. Then, slides were incubated for 10 min in $0.3 \%$ hydrogen peroxide solution and blocked with $10 \%$ goat serum for $30 \mathrm{~min}$. The rabbit polyclonal antibody for KLC2 (Proteintech; 17668-1-AP) diluted at 1:25 was mixed with mouse anti-Ki-67 nuclear antigen monoclonal antibody (Zhong ShanGolden Bridge Biological Technology; ZM-0166) diluted at $1: 1200$ and incubated overnight at $4{ }^{\circ} \mathrm{C}$. Slides were washed three times in PBS and incubated for $40 \mathrm{~min}$ at room temperature with secondary antibody: anti-rabbit IgG AP (1:5000; Ebiogenes; ab136817) and anti-mouse IgG HRP (1:5000; Ebiogenes; ab136815). After washing three times with PBS, substrates were added starting with fuschin red followed by DAB. After colour development with DAB, slides were boiled for $5 \mathrm{~min}$ in an antigen retrieval citrate solution $(\mathrm{pH}=6.0)$ to remove previously attached antibodies and washed several minutes with PBS. Slides were counterstained with instant haematoxylin.

Statistical analysis. SPSS 13.0 software package was used for statistical analysis. All experiments were repeated three times independently at least and all data were expressed as the mean \pm standard deviation. The difference between groups were calculated using the two-tailed unpaired Student's $t$-test (qRTPCR, wound-healing, migrating or invading cell numbers and
Dual-Luciferase Reporter Assay) and all $P<0.05$ was considered statistically significant. The correlation between miR-125b and KLC2 expression was analysed by Spearman's correlation. Kaplan-Meier method and the log-rank test were used to estimate variable in prediction of the patient overall survival. Correlations of KLC2 expression with clinic-pathological parameters were assessed using $\chi^{2}$ test. Hazard ratio and prognostic factors were determined by univariate and multivariate analyses using Cox regression model.

\section{RESULTS}

KLC2 protein is overexpressed in NSCLC and associated with poor clinical outcome of elderly NSCLC. As shown in Figure 1A, the protein level of KLC2 significantly increased in human NSCLC cell lines compared with normal lung epithelial cell line (HBE). And we noticed that the level of KLC2 protein was much higher in A549, H460 and H1299 in comparison with SPC-A-1 and 95D. In addition, the capacity of cell migration and invasion is also higher in NSCLC cell lines with high KLC2 expression (Figure 1B and Supplementary Figure 1). On the tissue microarray, KLC2 expression was predominantly diffuse and cytoplasmic staining in tumour cells, whereas normal lung alveoli and interstitial tissue exhibited negative or weak expression of KLC2 (Figure 1C, upper panel). Double staining assays (Figure 1C, lower panel) further demonstrated that KLC2 was diffusely expressed in the cytoplasm (red colour) of tumour cells, in which Ki-67 expression was restricted to the nuclei (brown colour). Kinesin-1 light chain-2 was overexpressed in $67.1 \%$ human primary NSCLC tissues but only in $2.1 \%$ adjacent non-tumour lung tissues (Figures $1 \mathrm{C}, P<0.001$ ). Although KLC2 expression level was related to neither any clinicopathologic factor nor the overall survival of 140 patients (Supplementary Tables 1 and 2 and Supplementary Figure 2), KLC2 overexpression significantly portended inferior overall survival (Figures $1 D, P=0.026)$ in elderly NSCLC patients $(\geq 60$ years of age, 81 cases). In the multivariate model, high KLC2 expression remained to be an independent negative predictor of prognosis for elderly NSCLC patients (Table 1). Notably, high KLC2 expression appeared to be associated with shorter overall survival in male patients, or in patients either with squamous cell carcinoma, or with N0 disease, or with Stage I/II disease, although the correlation did not reach statistical significance (Supplementary Figure 3). Taken together, these results suggest that KLC2 may have an important role in NSCLC progression.

KLC2 contributes to NSCLC cells invasion and migration in vitro. To characterize the function of KLC2 in NSCLC cells, both gain- and loss-of-function models were established with a transient transfection strategy. Successful overexpression and

Table 1. Univariate and multivariate analyses of individual parameters for correlations with survival rate: Cox proportional hazards model

\begin{tabular}{|c|c|c|c|c|c|c|}
\hline \multirow[b]{2}{*}{ Parameters } & \multicolumn{3}{|c|}{ Univariate } & \multicolumn{3}{|c|}{ Multivariate } \\
\hline & $P$ & HR & $95 \% \mathrm{Cl}$ & $P$ & HR & $95 \% \mathrm{Cl}$ \\
\hline Gender & 0.617 & 1.184 & $0.611-2.293$ & & & \\
\hline Histological differentiation & 0.769 & 1.112 & $0.549-2.250$ & & & \\
\hline Local advance & 0.629 & 1.189 & $0.589-2.403$ & & & \\
\hline Lymph node metastasis & 0.098 & 1.689 & $0.907-3.144$ & & & \\
\hline Histopathologic type & 0.886 & 0.957 & $0.529-1.733$ & & & \\
\hline KLC2 expression & 0.030 & 2.135 & $1.075-4.240$ & 0.029 & 2.329 & $1.091-4.972$ \\
\hline Clinical stage & 0.041 & 1.465 & $1.015-2.115$ & 0.113 & 1.341 & $0.933-1.926$ \\
\hline
\end{tabular}


knockdown of KLC2 was illustrated by western blotting and $\mathrm{qRT}$ PCR (Figure 2A). We investigated cell growth by MTT assay and found that KLC2 overexpression or knockdown produced no effects on lung cancer cells proliferation (Supplementary Figure 4). Next, we performed wound-healing assay and Transwell assays with or without Matrigel to evaluate whether KLC2 can affect NSCLC cells migration and invasion. The invasion and migration ability of SPC-A-1 and 95D cells ectopically expressing KLC2 were significantly enhanced when compared with control cells. Conversely, downregulation of KLC2 impaired the invasion and migration ability of NSCLC cells (Figure 2B and C). Therefore, these data further implied the biological importance of KLC2 upregulation in lung cancer development.
miR-125b regulates the expression of $\mathrm{KLC} 2$ at the translational level. Bioinformatic analysis implied that the $3^{\prime} \mathrm{UTR}$ region of KLC2 contains putative binding sites for miR-125b (Figure 3A). To determine whether miR-125b directly targets the $3^{\prime} \mathrm{UTR}$ of KLC2, we performed luciferase reporter assay by cloning the WT or MUT $3^{\prime}$ UTR of KLC2 into the downstream region of a luciferase reporter gene. The WT or MUT vector was co-transfected into HEK-293T and SPC-A-1 cells with miR-125b mimics or miR-control. We found that overexpression of miR-125b resulted in a two-fold decrease of luciferase activity of WT KLC2 $3^{\prime} \mathrm{UTR}$, as compared with the miR-control. Whereas the reduction of the luciferase activity with MUT KLC2 $3^{\prime}$ UTR was not observed (Figure 3B). Notably, analysis of the miR-125b expression levels in five human
A

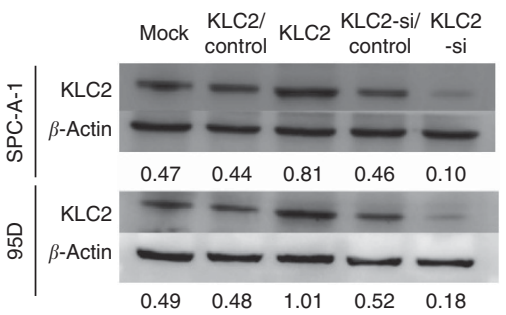

B

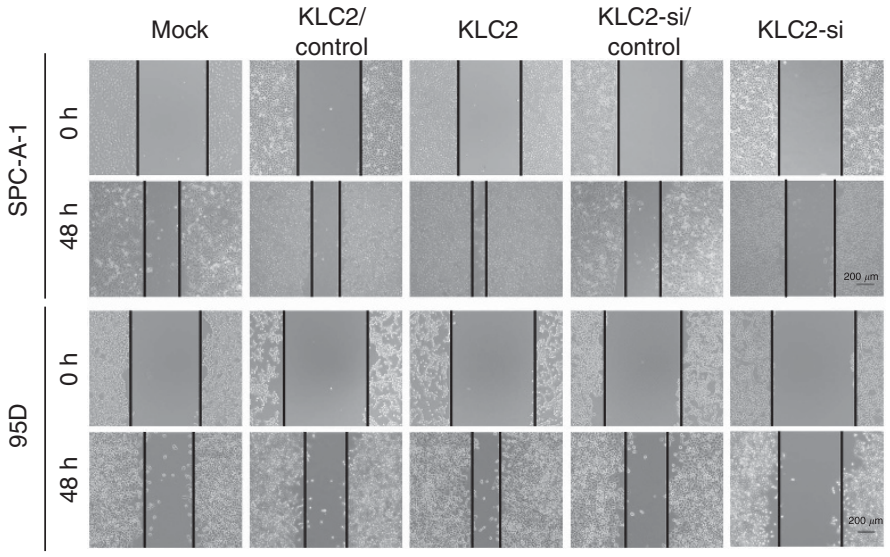

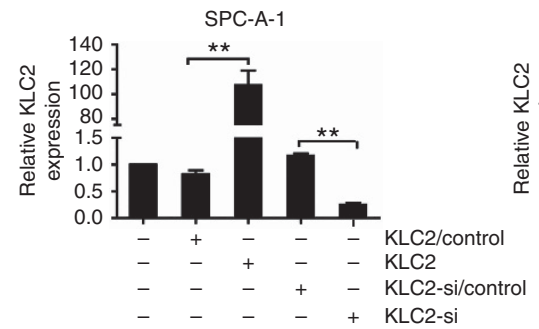
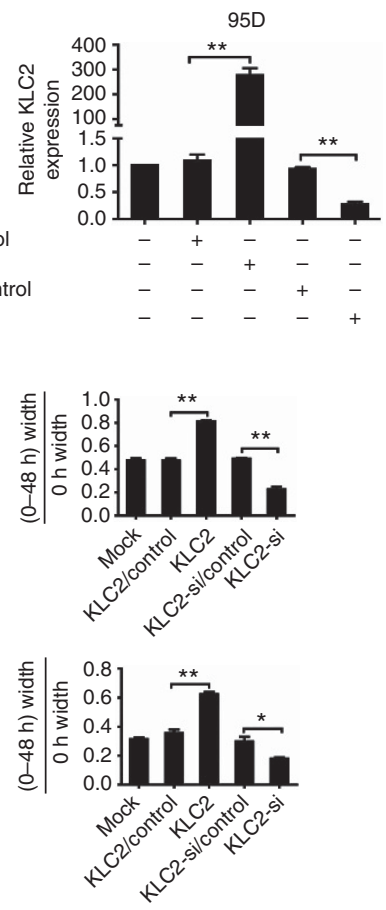

C

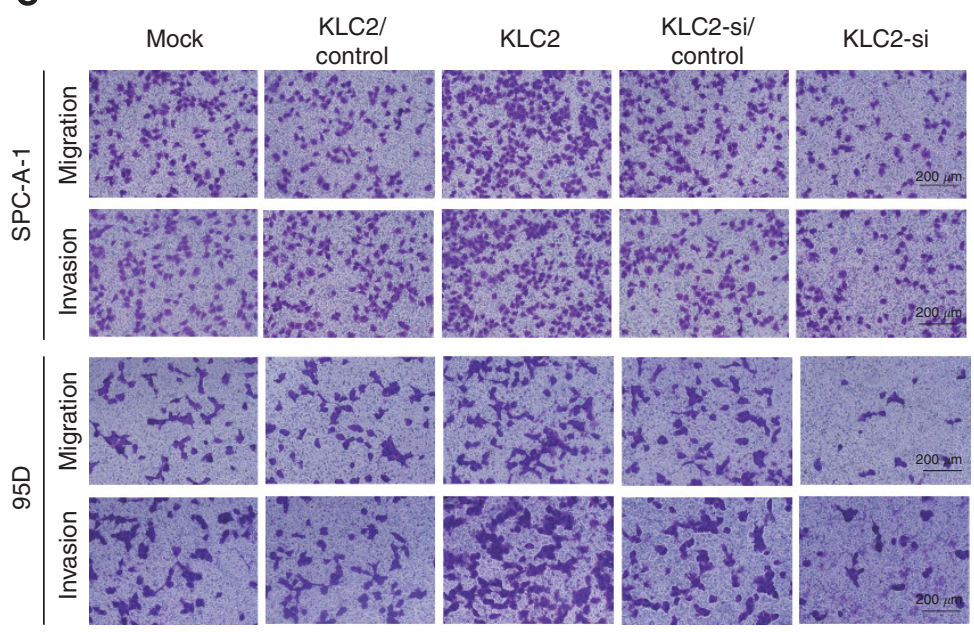

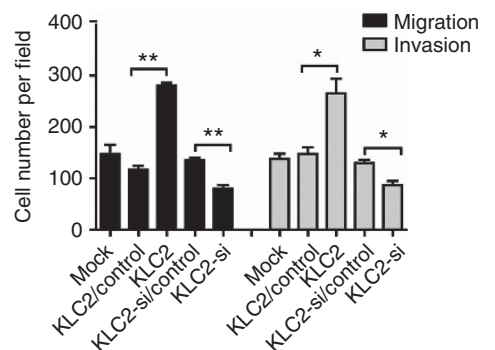

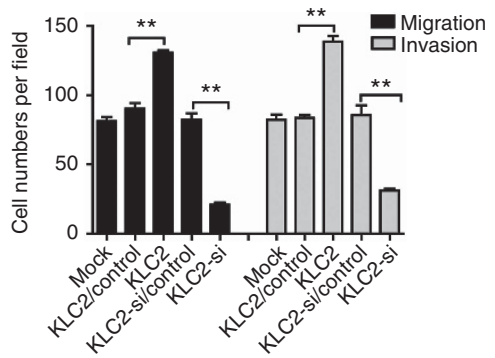

Figure 2. KLC2 contributes to NSCLC cells invasion and migration in vitro. (A) The endogenous KLC2 expression levels were illustrated by western blotting and qRT-PCR in SPC-A-1 and 95D cells transfected with KLC2-overexpressing plasmid, KLC2-si plasmid or their controls for $48 \mathrm{~h}$. (B) Wound-healing assay showed that overexpression of KLC2 stimulated, whereas inhibition of KLC2 suppressed SPC-A-1 and 95D cell migration. (C) Representative images (left, $\times 200$ ) of Transwell assays with or without a Matrigel and quantification (right) of cell migration and invasion. The migrated or invaded cells were counted from five random fields at $\times 200$ magnification $\left({ }^{\star} P<0.05,{ }^{\star \star} P<0.01\right.$ compared with controls). 
A

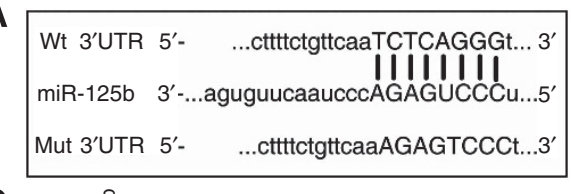

C

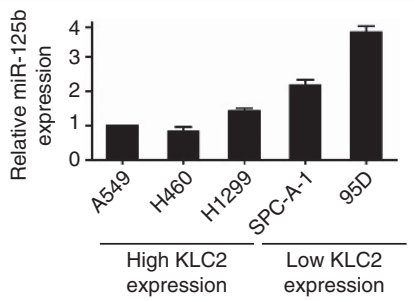

D

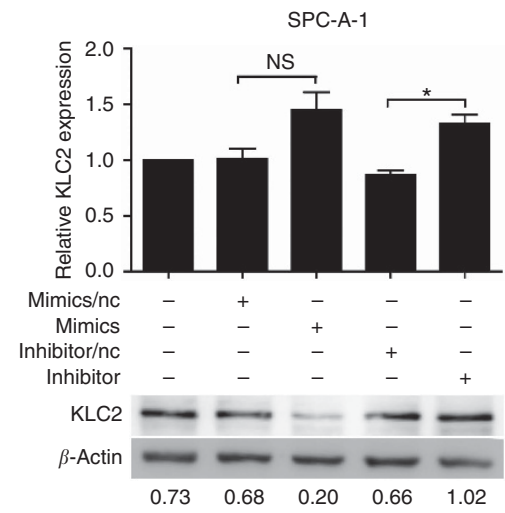

$\mathbf{E}$
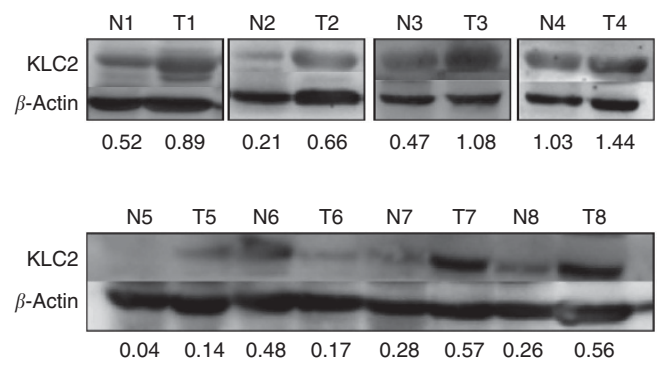

B
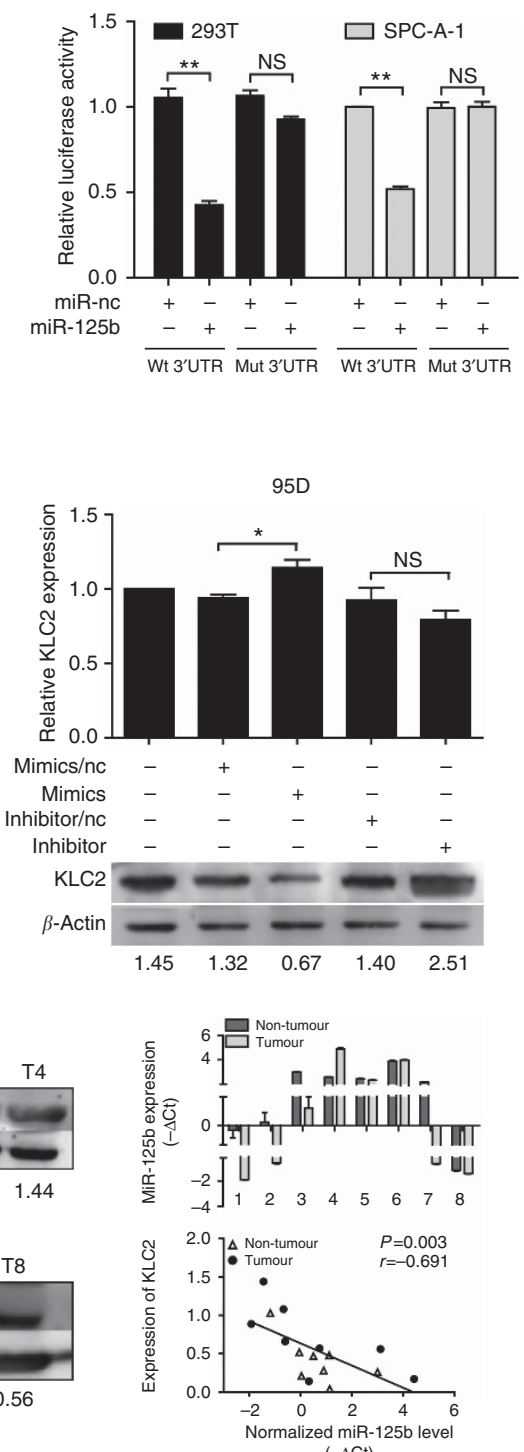

$(-\Delta \mathrm{Ct})$

Figure 3. KLC2 is the target of miR-125b. (A) Wild-type (Wt) and mutated (Mut) putative miR-125b-binding sequence in the $3^{\prime} U T R$ of KLC2 gene. (B) Verification of interactions between miR-125b and the 3'UTR of KLC2 in HEK-293T and SPC-A-1 cells by luciferase reporter assay. The data are presented as the mean \pm s.d. from three independent experiments. (C) Real-time PCR analysis to quantify the endogenous levels of miR-125b in five NSCLC cells. U6 was used as a control. (D) MiR-125b negatively regulates KLC2 expression at post-transcriptional level. (E) The inverse correlation between miR-125b and KLC2 protein expression in eight pairs of clinical human NSCLC and non-tumorous lung tissues $(r=-0.691$, $P=0.003)$. The $P$ values were calculated by two-tailed Student's t-test based on three independent experiments $\left({ }^{\star} P<0.05,{ }^{\star \star} P<0.01\right)$.

NS, non-significant.

NSCLC cell lines showed that miR-125b was markedly suppressed in cells with high KLC2 expression (A549, H460 and H1299) compared with cells with low KLC2 expression (SPC-A-1 and 95D; Figure $3 \mathrm{C}$ ). To further confirm the regulatory effect of miR-125b on KLC2 expression, we measured the mRNA and protein level of KLC2 in SPC-A-1 and 95D cells after ectopically expressing or suppressing miR-125b. Data (Figure 3D) showed that a 1- to 2.4fold decrease of KLC2 protein expression in response to miR-125b upregulation was observed. Conversely, a corresponding increment in protein expression for KLC2 was also demonstrated following transfection with the miR-125b inhibitor in SPC-A-1 and 95D cells. But miR-125b had less (no more than 40\%) effect on KLC2 mRNA expression (Figure 3D). Moreover, KLC2 protein and miR-125b expression were inversely correlated in our clinical NSCLC and non-tumorous lung samples $(R=-0.691, P=0.003$; Figure $3 \mathrm{E})$. Taken together, these data suggested that miR-125b repressed KLC2 expression at protein level by targeting the $3^{\prime} \mathrm{UTR}$ of KLC2.

KLC2 has a critical role in miR-125b-mediated invasion and migration of NSCLC cells. To determine whether KLC2 was critical mediators of miR-125b function in NSCLC invasion and migration, KLC2 was overexpressed in miR-125b-overexpressing SPC-A-1 and 95D cells. The transfection efficiency was verified by qRT-PCR and western blotting (Figure 4A). The effect of ectopic miR-125b upregulation on KLC2 expression were further confirmed to be consistent with above results at both mRNA and protein levels. However, overexpression of KLC2 did not lead to any change in miR-125b expression.

Cells were then analysed for cell invasion and migration. As expected, ectopic expression of miR-125b significantly reduced migration and invasion potential of NSCLC cells, which was 
A
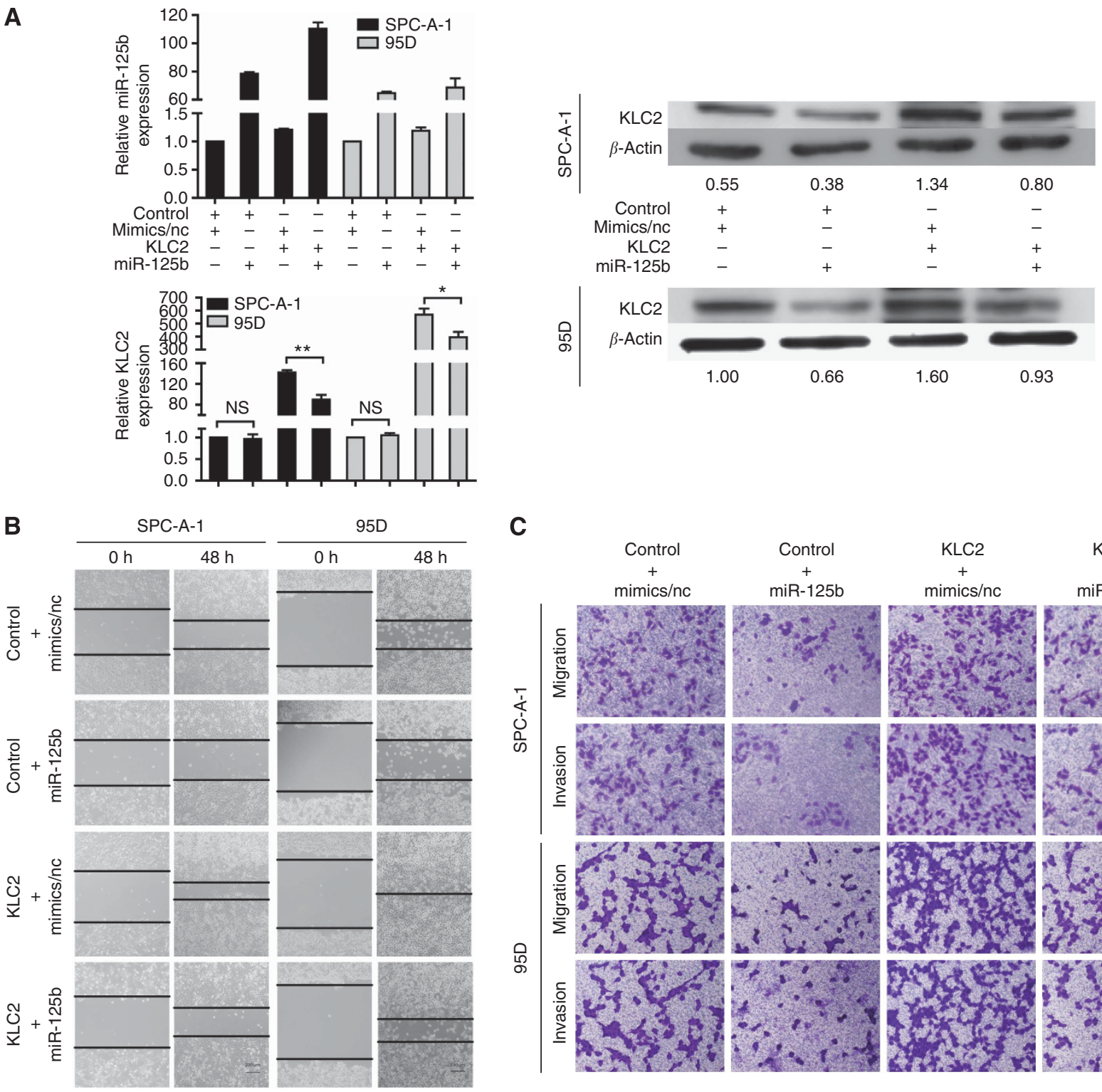

C

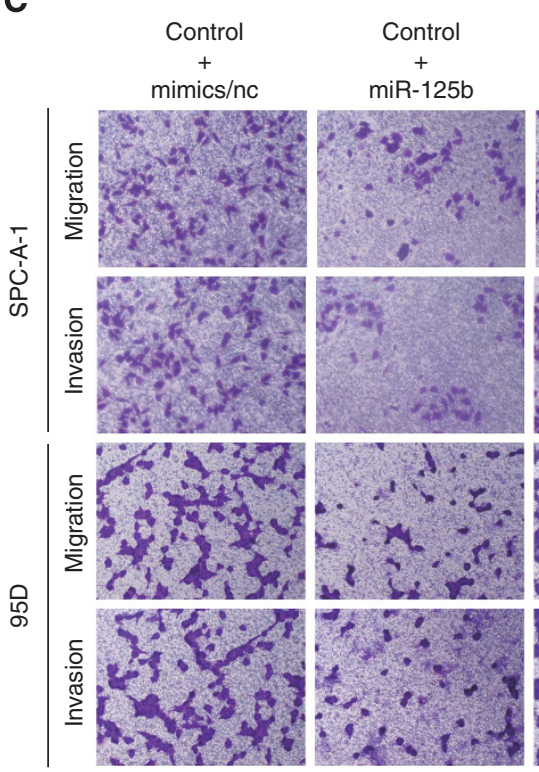

SPC-A-1

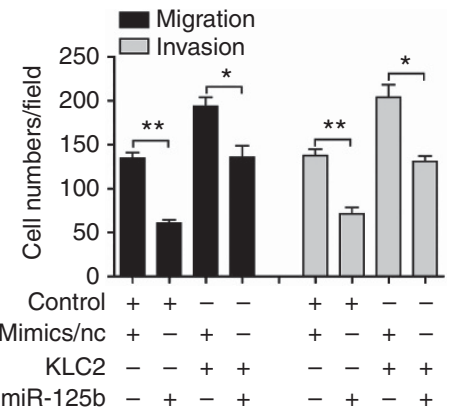

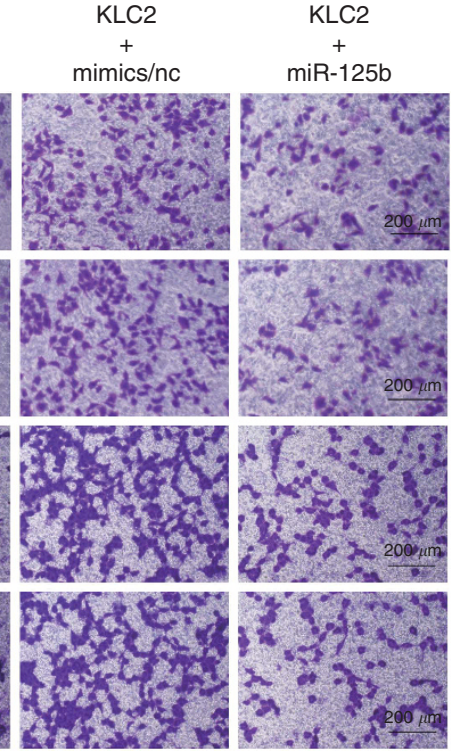

95D

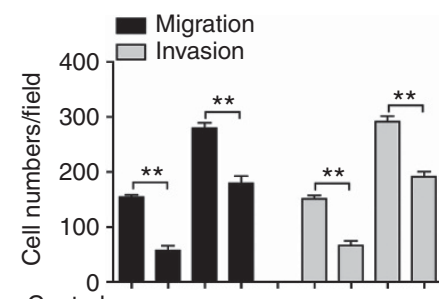

Control ++--++-Mimics/nc +-+-+++ $\mathrm{KLC} 2--++-++$ miR-125b -+++-+++

Figure 4. Reintroduction of KLC2 reversed miR-125b-induced retardation of migration and invasion of lung cancer cells. (A) The expression of miR-125b, KLC2 mRNA and protein in NSCLC cells co-transfected with KLC2-overexpressing plasmid and miR-125b mimics or controls.

(B) Wound-healing assay and (C) Transwell assays were performed to confirm that ectopic expression of KLC2 rescued the inhibition of cell migration and invasion associated with the re-expression of miR-125b $\left({ }^{\star} P<0.05,{ }^{\star \star} P<0.01\right)$. NS, non-significant.

contrary to the promotive effect of KLC2 on NSCLC cells. Most important, exogenously expressed KLC2 almost completely reversed the inhibitory effect of miR-125b on migration and invasion in both SPC-A-1 and 95D cells (Figure 4B and C). Therefore, KLC2 was an important mediator responsible for the effects of miR-125b on NSCLC cell migration and invasion.

\section{DISCUSSION}

The kinesin superfamily (KIF) is a group of proteins that act as molecular motor and generate ATP-dependent movement of vesicles and organelles along microtubules, which is classified into 
14 families. Increasing evidence (Liu et al, 2013) indicates that aberrant expression of kinesin proteins in cancers have been studied as diagnostic or prognostic indicators. Some kinesin proteins are involved in the malignant phenotype as well as Taxane resistance of solid tumour (Liu et al, 2013). As one light chain of kinesin-1, KLC2 is directly involved in the binding and release of cargos (Morfini et al, 2002), one of which is Smad2 (Batut et al, 2007). Recently, Manser et al (2012) reported that lemur tyrosine kinase- 2 might impact upon prostate cancer by promoting binding of Smad2 to KLC2. However, the expression pattern of KLC2 in tumour tissues, the potential clinicopathological implications of altered KLC2 expression, as well as the functions of KLC2 in malignant phenotype of NSCLC cells have not been studied.

Our study is the first publication to analyse KLC2 expression in NSCLC patients. The results showed that KLC2 protein expression level in NSCLC tissues was significantly higher than that in adjacent non-neoplastic lung tissues. Furthermore, we found that high KLC2 expression led to shorter survival in elderly patients with NSCLC, and multivariate analysis indicated the negative prognostic value of KLC2 in clinic. We also provided insights about the biological effects of KLC2 in the malignance of NSCLC. Our data provided the first evidence that the expression of KLC2 was correlated with the invasion and migration of NSCLC cell lines in vitro. Together, these findings suggested the oncogenic role of KLC2 in NSCLC development.

It has been reported (Lewis et al, 2005) that well over one-third of human genes appeared to be conserved miRNA targets, thereby suggesting pivotal roles of miRNAs in physiological and pathological processes, including human carcinogenesis. MiR-125b is a ubiquitously and aberrantly expressed miRNA in a broad variety of tumours. Mature miR-125b is generated from miR-125b-1 and miR-125b-2, both of which reside in the commonly deleted regions in human lung cancer based on genome scanning data (Martin et al, 2003; Yamada et al, 2008). A miRNA expression signature also identified miR-125b as downregulated miRNA in squamous cell lung carcinoma tissues compared with normal tissues (Gao et al, 2011). Cell biology experiments further revealed that miR$125 \mathrm{~b}$ expression not only induced spontaneous apoptosis in lung cell line, but also sensitised cancer cells to diverse apoptotic stimuli (Gong et al, 2013). In our previous study (Li et al, 2013), we demonstrated that inhibition of miR-125b promoted the migration and invasion of NSCLC cells in vitro. All the above supported the idea that miR-125b could serve as a tumour suppressor in the development of NSCLC. However, the target genes elucidating the role of miR-125b in NSCLC development have not been identified.

In this study, we identified KLC2 as putative miR-125b target gene using three bioinformatics tools. Our data demonstrated that the restoration of miR-125b expression in lung cancer cells led to a significant decrease at the level of KLC2 protein but not of KLC2 mRNA; conversely, the inhibition of miR-125b further upregulated KLC2 protein expression. Luciferase reporter gene experiments showed that miR-125b directly targets the $3^{\prime} \mathrm{UTR}$ of KLC2. Furthermore, we found miR-125b high-expression NSCLC specimens tended to express low levels of KLC2. Last, our data indicated that the overexpression of KLC2 almost completely antagonised the miR-125b restoration-induced inhibition of cancer invasion and migration. Therefore, these data suggested that KLC2 functioned as a key mediator responsible for the effects of miR$125 \mathrm{~b}$ on NSCLC cell invasion and migration.

In summary, we identified KLC2 as a novel target and functional mediator of miR-125b in NSCLC and revealed the post-transcriptional mechanism by which miR-125b might regulate KLC2 expression. We also provided the first evidence to determine the oncogenic potential of KLC2 in the progression of NSCLC by combining clinical and experimental studies. Our work provides new insights about the molecular mechanisms underlying NSCLC carcinogenesis and potential targets for future therapeutic invention. However, a further detailed study is needed to elucidate the downstream mechanism of KLC2 in NSCLC.

\section{ACKNOWLEDGEMENTS}

The present work was supported by grants from National Natural Science Foundation of China (81001047, 81071827), Science and technology projects in Guangdong Province (2012B031800127), Excellent Young Teachers Program of Higher Education of Guangdong Province (Yq2013040), Research Fund for the Science and Technology Star of Zhujiang of Guangzhou City (2014J2200031).

\section{CONFLICT OF INTEREST}

The authors declare no conflict of interest.

\section{REFERENCES}

Akhurst RJ, Hata A (2012) Targeting the TGF $\beta$ signalling pathway in disease. Nat Rev Drug Discov 11(10): 790-811.

Batut J, Howell M, Hill CS (2007) Kinesin-mediated transport of Smad2 is required for signaling in response to TGF-beta ligands. Dev Cell 12(2): 261-274.

Fan DN, Tsang FH, Tam AH, Au SL, Wong CC, Wei L, Lee JM, He X, Ng IO, Wong CM (2013) Histone lysine methyltransferase, suppressor of variegation 3-9 homolog 1, promotes hepatocellular carcinoma progression and is negatively regulated by microRNA-125b. Hepatology 57(2): 637-647.

Ferlay J, Soerjomataram I, Ervik M, Dikshit R, Eser S, Mathers C, Rebelo M, Parkin DM, Forman D, Bray F (2013) GLOBOCAN 2012 v1.0, Cancer Incidence and Mortality Worldwide: IARC CancerBase No. 11 [Internet]. International Agency for Research on Cancer: Lyon, France, Available at http://globocan.iarc.fr.

Gao W, Shen H, Liu L, Xu J, Xu J, Shu Y (2011) MiR-21 overexpression in human primary squamous cell lung carcinoma is associated with poor patient prognosis. J Cancer Res Clin Oncol 137(4): 557-566.

Gong J, Zhang JP, Li B, Zeng C, You K, Chen MX, Yuan Y, Zhuang SM (2013) MicroRNA-125b promotes apoptosis by regulating the expression of Mcl-1, Bcl-w and IL-6 R. Oncogene 32(25): 3071-3079.

Hirokawa N, Noda Y, Tanaka Y, Niwa S (2009) Kinesin superfamily motor proteins and intracellular transport. Nat Rev Mol Cell Biol 10(10): 682-696.

Jiang F, Liu T, He Y, Yan Q, Chen X, Wang H, Wan X (2011) MiR-125b promotes proliferation and migration of type II endometrial carcinoma cells through targeting TP53INP1 tumor suppressor in vitro and in vivo. BMC Cancer 11: 425.

Kappelmann M, Kuphal S, Meister G, Vardimon L, Bosserhoff AK (2013) MicroRNA miR-125b controls melanoma progression by direct regulation of c-Jun protein expression. Oncogene 32(24): 2984-2991.

Lewis BP, Burge CB, Bartel DP (2005) Conserved seed pairing, often flanked by adenosines, indicates that thousands of human genes are microRNA targets. Cell 120(1): 15-20.

Li J, You T, Jing J (2014) MiR-125b inhibits cell biological progression of Ewing's sarcoma by suppressing the PI3K/Akt signalling pathway. Cell Prolif 47(2): 152-160.

Li Y, Chao Y, Fang Y, Wang J, Wang M, Zhang H, Ying M, Zhu X, Wang H (2013) MTA1 promotes the invasion and migration of non-small cell lung cancer cells by downregulating miR-125b. J Exp Clin Cancer Res 32: 33 .

Liang L, Wong CM, Ying Q, Fan DN, Huang S, Ding J, Yao J, Yan M, Li J, Yao M, Ng IO, He X (2010) MicroRNA-125b suppressesed human liver cancer cell proliferation and metastasis by directly targeting oncogene LIN28B2. Hepatology 52(5): 1731-1740.

Liu X, Gong H, Huang K (2013) Oncogenic role of kinesin proteins and targeting kinesin therapy. Cancer Sci 104(6): 651-656.

Manser C, Guillot F, Vagnoni A, Davies J, Lau KF, McLoughlin DM, De Vos KJ, Miller CC (2012) Lemur tyrosine kinase-2 signalling regulates 
kinesin-1 light chain-2 phosphorylation and binding of Smad2 cargo. Oncogene 31(22): 2773-2782.

Martin ES, Cesari R, Pentimalli F, Yoder K, Fishel R, Himelstein AL, Martin SE, Godwin AK, Negrini M, Croce CM (2003) The BCSC-1 locus at chromosome 11q23-q24 is a candidate tumor suppressor gene. Proc Natl Acad Sci USA 100(20): 11517-11522.

Morfini G, Szebenyi G, Elluru R, Ratner N, Brady ST (2002) Glycogen synthase kinase 3 phosphorylates kinesin light chains and negatively regulates kinesin-based motility. EMBO J 21(3): 281-293.

Tang F, Zhang R, He Y, Zou M, Guo L, Xi T (2012) MicroRNA-125b induces metastasis by targeting STARD13 in MCF-7 and MDA-MB-231 breast cancer cells. PLoS One 7(5): e35435.

Xu N, Zhang L, Meisgen F, Harada M, Heilborn J, Homey B, Grandér D, Ståhle M, Sonkoly E, Pivarcsi A (2012) MicroRNA-125b down-regulates matrix metallopeptidase 13 and inhibits cutaneous squamous cell carcinoma cell proliferation, migration, and invasion. J Biol Chem 287(35): 29899-29908.

Yamada H, Yanagisawa K, Tokumaru S, Taguchi A, Nimura Y, Osada H, Nagino M, Takahashi T (2008) Detailed characterization of

a homozygously deleted region corresponding to a candidate tumor suppressor locus at 21q11-21 in human lung cancer. Genes Chromosomes Cancer 47(9): 810-818.

Yuxia M, Zhennan T, Wei Z (2012) Circulating miR-125b is a novel biomarker for screening non- small-cell lung cancer and predicts poor prognosis. J Cancer Res Clin Oncol 138(12): 2045-2050.

Zhang J, Lu L, Xiong Y, Qin W, Zhang Y, Qian Y, Jiang H, Liu W (2014) MLK3 promotes melanoma proliferation and invasion and is a target of microRNA-125b. Clin Exp Dermatol 39(3): 376-384.

Zhu X, Guo Y, Li X, Ding Y, Chen L (2010) Metastasis-associated protein 1 nuclear expression is associated with tumor progression and clinical outcome in patients with non-small cell lung cancer. J Thorac Oncol 5(8): 1159-1166.

This work is published under the standard license to publish agreement. After 12 months the work will become freely available and the license terms will switch to a Creative Commons AttributionNonCommercial-Share Alike 4.0 Unported License.

Supplementary Information accompanies this paper on British Journal of Cancer website (http://www.nature.com/bjc) 